\title{
Isobar Channels in the Production of $\pi+\pi$-Pairs on a Proton by Virtual Photons
}

\author{
V. D. Burkert, V. I. Mokeev, N. V. Shvedunov, A. A. Boluchevskii), M. Battaglieri, E. N. \\ Golovach, L. Elouardrhiri, K. Joo, E. L. Isupov, B. S. Ishkhanov, N. S.Markov, M.Ripani, J. \\ Ricco, V. V. Sapunenko, M. Taiuti, and G. V. Fedotov
}

\begin{abstract}
A new approach is developed for evaluating contributions of various isobar channels to the double chargedpion production in the reactions $\gamma r, v p \rightarrow \pi+\pi-p$. This approach makes it possible to determine both respective cross sections and amplitudes for quasi-two-particle channels from a fit to data on cross sections for a three-particle final state. The diffractive ansatz, which is widely used to describe rho-meson production, is modified to meet the purpose of the description of data in the near-threshold and subthreshold regions. The cross sections for rho-meson electroproduction in the energy region of nucleon-resonance excitation are determined for the first time on the basis of the latest data of the CLAS Collaboration. PACS numbers: 13.40.Gp
\end{abstract}

\section{INTRODUCTION}

A vast body of experimental data on the production of $\pi^{+} \pi^{-}$pairs on a proton by real and virtual photons has been accumulated to date within the program for studying the structure of nucleon

resonances $(N *)$ at the Thomas Jefferson National Accelerator Facility (TJNAF, USA) [1-4]. This channel is of particular interest for studying nucleon resonance physics, since the decay of the majority of excited nucleon states having amass above $1.65 \mathrm{GeV}$ involves predominantly the emission of pion pairs. Investigations of nucleon resonances in the exclusive channel of double pion electroproduction furnished important physics results. Signals from a possible new type of nucleon excitations, the so-called $3 / 2^{+}(1720)$ missing-baryon state, were discovered for the first time [3].Detailed investigations of nonresonance mechanisms of photo- and electroproduction of $\pi^{+} \pi^{-}$pairs on a proton are being performed at the present time. A phenomenological theory for describing the double charged-pion production on a proton by real and virtual photons was developed. The original version of this model was used to analyze CLAS data on double pion electroproduction [3]. A detailed description of this approach was given in [5-8]. The latest results that emerged from the development of the phenomenological model in question are given in [1, 2]. At the present time, this approach is being used to analyze data of the CLAS Collaboration [3, 4] on double charged-pion photo- and electroproduction. The objective of this analysis is to determine the $Q 2$ dependences of electromagnetic form factors for the majority of nucleon resonances that are characterized by masses below $2.0 \mathrm{GeV}$ and which are excited on a proton by real and virtual photons at virtualities $Q 2$ between 0 and $1.5 \mathrm{GeV}^{2}$.

Investigation of double charged-pion production on a proton by real and virtual photons also provides important information about the contributions of various isobar channels to the reaction $\gamma r, v p \rightarrow \pi+\pi-p$. Data on isobar channels are of great interest for studying non-perturbative stronginteraction mechanisms involving both resonance and nonresonance processes. The approach developed in [9] to describe strong interactions in the non-perturbative region establishes relations between amplitudes and cross sections for various isobar channels. This approach relies on fundamental principles of QCD and relates them to nonperturbative dynamics within $1 / N_{c}$ expansion. Data on cross sections and amplitudes for various isobar channels form an important input for testing adequacy of the description of strong interaction dynamics in the nonperturbative region within approaches based on expansions at large $N_{c}$. Such data are also of importance for performing a coupled-channel analysis [10] of reactions that involve hadrons and photons and in which isobar channels of double pion photoproduction lead to asymptotic final states.

Information about contributions of various isobar channels is also of importance for describing coupling to open inelastic channels in the $\pi+\pi-p$ three particle final state. The inclusion of coupling to open inelastic channels is mandatory in analyzing reactions involving either photons or hadrons in the case where the invariant mass of the final hadron system lies in the region $W$ $>1.6 \mathrm{GeV}$. 
(a)

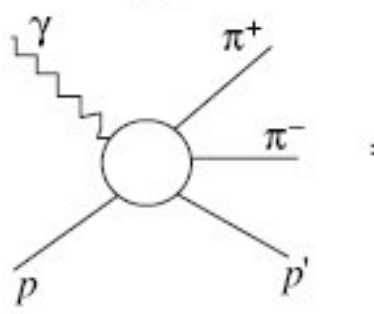

(c) (b)

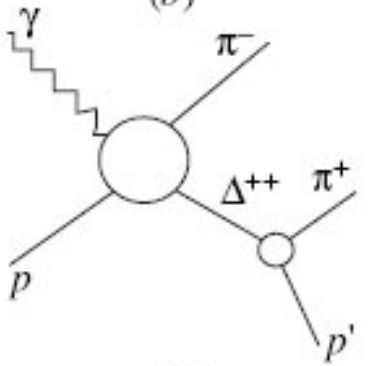

(d)

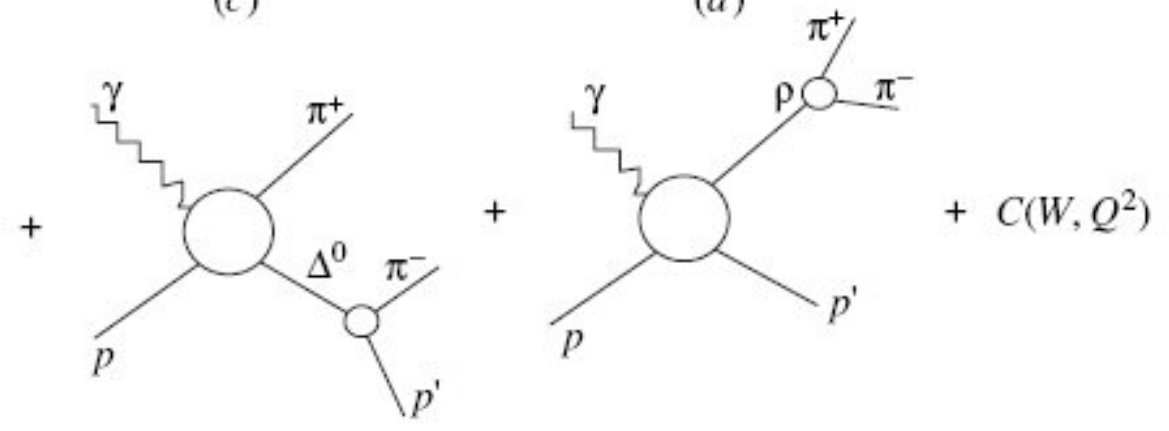

Fig. 1. [6] Mechanisms of the reaction $y p \rightarrow \pi+\pi-p$ in the dynamical model considered in [5-8]. Here, $C\left(W, Q^{2}\right)$ is the three-particle phase space.

In this kinematical region, the number of open inelastic channels grows significantly with increasing $W$, so that a consistent inclusion of channel-coupling effects becomes crucial for reliably extracting information about nonperturbative strong-interaction mechanisms being studied. In the present study, we develop a procedure for separating the isobar channels

$$
\begin{gathered}
\gamma r, v p \rightarrow \pi-\Delta++,(1) \\
\gamma r, v p \rightarrow \pi+\Delta 0,(2) \\
\gamma r, v p \rightarrow \rho p,(3)
\end{gathered}
$$

which contribute to the production of $\pi^{+} \pi^{-}$pairs by real and virtual photons.

The production of $\pi^{+} \pi^{-}$pairs by photons is described on the basis of the dynamical model that was developed in [5-8] and whose phenomenological parameters were determined from a fit to measured cross sections. The contributions of the channels in (1)-(3) are calculated by using the parameter values fixed in this way.

These contributions can be determined both at the level of integrated and differential cross sections and at the level of helicity amplitudes. In this article, we present the results obtained at the first stage of investigations into contributions from the isobar channels (1)-(3) to the reactions $\gamma r, v p \rightarrow \pi+\pi-p$. The main objective was to extract information about integrated cross sections for the channel in (3) from data of the CLAS Collaboration on double charged-pion electroproduction [3]. These CLAS data make it possible to determine, for the first time, the cross sections and amplitudes for this channel in the nucleon-resonance-mass region $W<2.1 \mathrm{GeV}$, which covers the near-threshold and sub-threshold regions.

The results of the CLAS Collaboration that concern cross sections for double charged-pion electroproduction on a proton [3] are unique worldwide and form a set of detailed data on various types of single differential cross sections. Invariant-mass distributions of $\pi^{+} \pi^{-}$and $\pi^{+} p$ finalparticle pairs and the angular distributions of negatively charged pions in the c.m. frame were fitted simultaneously in each of the measured intervals of $W$ and $Q^{2}$. 
(a)

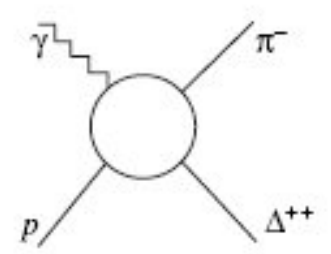

(b)

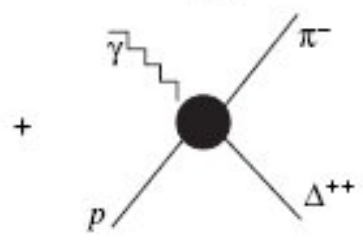

(d)

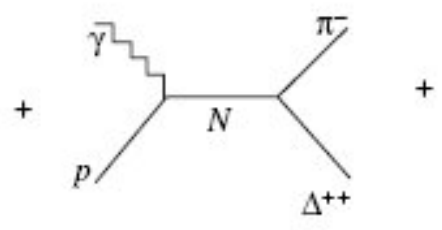

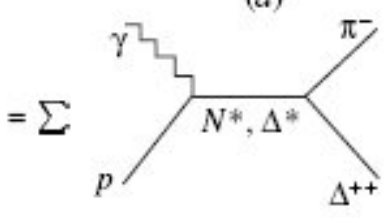

(c)

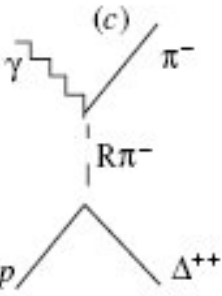

(e)

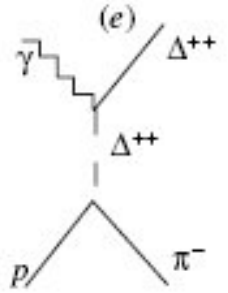

$(f)$
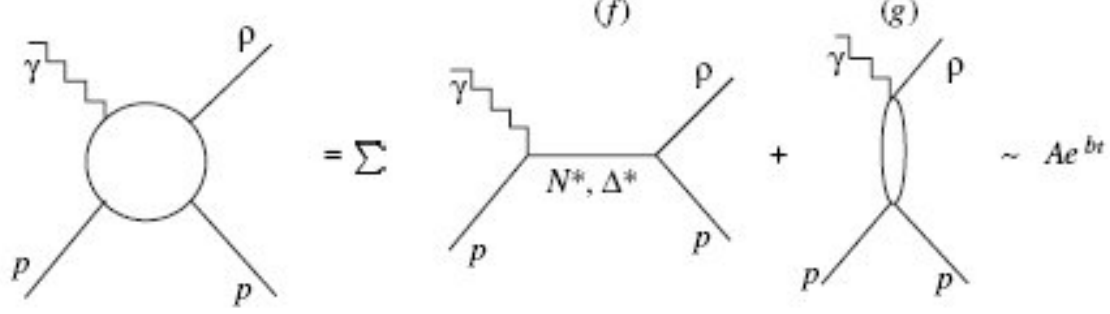

Fig. 2. [5] Tree diagrams describing the quasi-two-particle channels (1)-(3) within the model considered in [5-8]: $(a, f)$ contributions from $N_{*}$ and $\Delta *,(b-e)$ minimum set of gauge-invariant Born terms where singlepion exchange is Reggeized, and $(g)$ diffractive rho-meson production.

The database of observables measured in all previously performed experiments is much inferior to the CLAS data both in the number of measured quantities and in resolution. Thus, an analysis of the CLAS data would make it possible to obtain the most reliable information about the contributions of various isobar channels.

\section{METHOD FOR DETERMINING ISOBAR-CHANNEL CONTRIBUTIONS TO THE REACTIONS $\gamma r, v p \rightarrow \pi+\pi-p$}

The cross sections for the isobar channels (1)-(3) can be determined from a fit to the entire body of the measured differential cross sections for the reactions $\gamma r, v p \rightarrow \pi+\pi-p$ on the basis of the dynamical model developed in [5-8].

This dynamical model describes the total amplitude for the production of the $\pi+\pi-p$ final state as a superposition of the isobar channels (1)-(3), which involve the formation of unstable isobars in intermediate states and their subsequent decay to the final three-particle system $\pi+\pi-p$. The diagrams corresponding to the isobar channels (1)-(3) are shown in Fig. 1. The amplitudes of these diagrams are calculated under the assumption of a Breit-Wigner factorization into the amplitude for the production of a quasi-two-particle intermediate state and the amplitude for the subsequent decay of the unstable intermediate particle to final products. The propagation of an 
unstable isobar is described by a Breit-Wigner propagator whose total width is dependent on the invariant mass of decay products. A procedure that can be used to calculate the three-particle amplitudes for the diagrams in Fig. 1 are described in detail elsewhere $[6,7]$.

A comparison of the world-average data obtained previously $[11,12]$ and the latest data of the CLAS Collaboration $[3,4]$ on
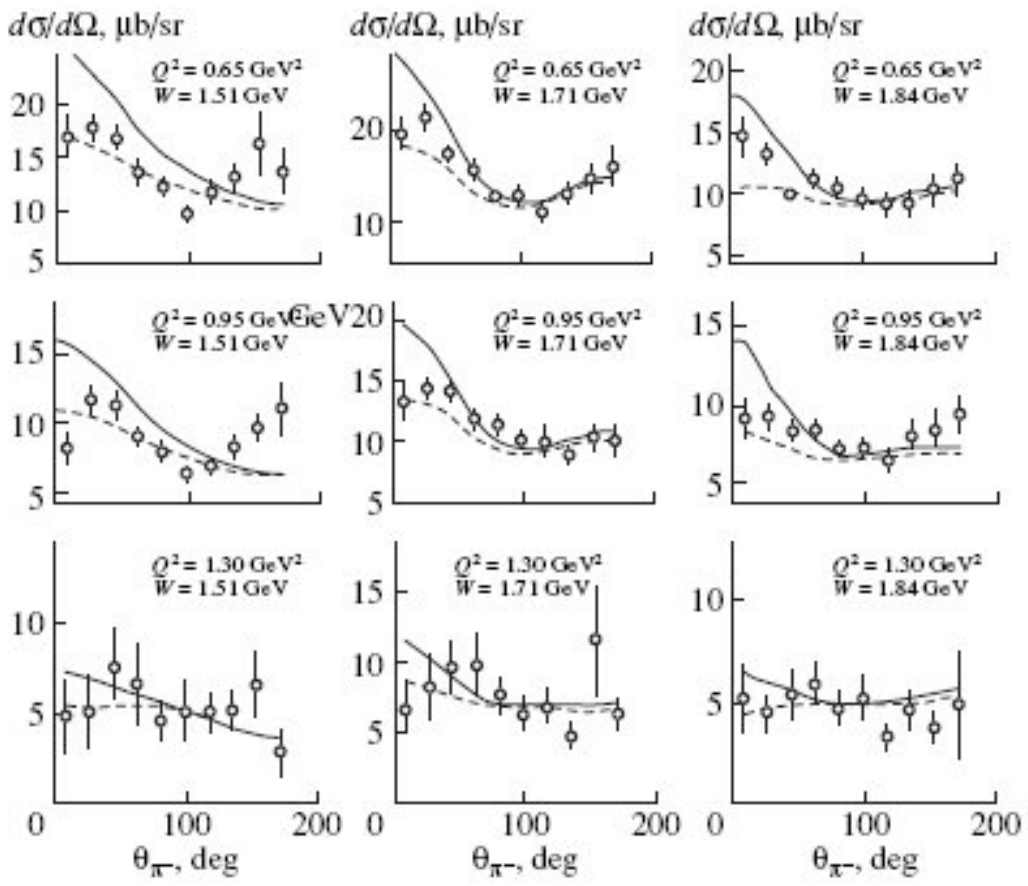

Fig. 3. Angular distributions of negatively charged pions in the c.m. frame at $\mathrm{Q}^{2}=$ (upper row) 0.65 , (middle row) 0.95 , and (lower row) $1.30 \mathrm{GeV}^{2}$ and at $W=$ (left column) 1.51, (middle column) 1.71, and (right column) $1.84 \mathrm{GeV}$. Shown in the figure is the range of the scatter of the cross sections (region between the solid and dashed curves) that corresponds to the change in $\alpha_{\mathrm{R}}$ from 1.1 to 1.7 (see Section 3.1). The displayed points represent experimental data, while the dashed and solid curves correspond to the values calculated at $\alpha_{\mathrm{R}}=1.1$ and at $\alpha_{\mathrm{R}}=1.7$, respectively.

double charged-pion photo- and electroproduction on a proton with the results of the calculations performed in [5-7] revealed that the tree diagrams in Fig. 1 do not exhaust the entire set of mechanisms for this exclusive channel. Processes other than those represented by the diagrams in Fig. 1 (residual mechanisms) were parameterized in the approximation of three-particle phase space. The amplitude of the residual mechanisms satisfies the requirement of invariance under spatial inversion. This is ensured by the phase-space factor that was discussed in [13]. The absolute value of the three particle phase space is described by a smooth function $C\left(W, Q^{2}\right)$ that is dependent on the invariant mass of the final hadron system, $W$, and on the photon virtuality, $Q^{2}$. This function is determined from a fit to the entire set of differential cross sections measured over a region of final-hadron-system invariant masses and photon virtualities that is centered at $\left(W, Q^{2}\right)$. The widths of the ranges of the measurements in $W$ and $Q^{2}$ were $25 \mathrm{MeV}$ and $0.3 \mathrm{GeV}^{2}$, respectively.

Each of the quasi-two-particle mechanisms represented by the diagrams in Fig. 1 is described in terms of a set of nucleon-resonance excitations in the $s$ channel of photon-proton interaction and a set of nonresonance processes. The resonance and nonresonance mechanisms corresponding to the isobar channels (1)-(3) are illustrated in Fig. 2.

Resonance mechanisms are described in the Breit-Wigner approximation. The off-shell evolution of the amplitudes for the hadronic decays of nucleon resonances and the off-shell evolution of 
their total widths are taken into account by introducing centrifugal barrier factors. Details of the calculation of resonance amplitudes are given elsewhere $[5,6]$. All nucleon-resonance and deltaisobar excitations ( $N^{*}$ and $\Delta^{*}$, respectively) of four-star status from the Particle Data Group tables and mass below $2 \mathrm{GeV}$ that have observed decays to a final state featuring two pions were taken into account in the model used. The three-star states $D_{13}(1700)$ and $P_{11}(1710)$ were also included. Signals from the possible new state $3 / 2^{+}(1720)$ were found for the first time in analyzing CLAS data from [3]. This state was also included in the description of resonance mechanisms. The amplitudes for hadronic decays of nucleon resonances were calculated within the approach described in [5]. They were obtained from the $L S$ partial widths presented for $N^{*}$ and $\Delta^{*}$ decays through the $\pi \Delta$ and $\rho p$ channels in the analysis reported in [14] and renormalized to the total hadronic widths in the Particle Data Group tables. The $N^{*}$ electromagnetic form factors $A_{1 / 2}$ and $A_{2 / 3}$ were free parameters that were determined from a fit to the measured cross sections for the reactions $\gamma r, v p \rightarrow \pi+\pi-p$.

Nonresonance mechanisms for the quasi-two particle channels (1) and (2) (Figs. $2 b-2 e$ ) were described by a minimal set of gauge-invariant Born terms. Effects involving the exchange of the entire set of mesons associated with the pion Regge trajectory were taken into account by replacing the singlepion propagator in the $t$ channel (see Fig. 2c) by the respective Reggeized propagator, as was proposed in [15]. The nonresonance amplitudes for the isobar channels (1) and (2) were calculated in [5]. The effective coupling $\alpha_{\mathrm{R}}$ of the pion Regge trajectory to the $p \rightarrow \Delta$ transition current (Fig. 2c) is a free parameter for the nonresonance component of the mechanisms of reactions (1) and (2). The coupling constant $\alpha_{R}$ is assumed to be independent of the invariant mass of the final hadron system, $W$, and is determined from a fit to the entire set of measured differential cross sections for double charged-pion production. The nonresonance mechanisms in channel (3) were described in the diffraction approximation. The calculation of the respective amplitudes is given in $[8,16]$. The diffraction approximation is highly accurate in the Mandelstam variable range $t<0.5 \mathrm{GeV}^{2}$. In the energy region of nucleon-resonance excitations, only this range of the variable $t$ has an effect on the cross section for the reactions $\gamma r, v p \rightarrow \pi+\pi-p$ beyond the measurement uncertainties. In the region $t>0.5 \mathrm{GeV}^{2}$, nonresonance processes, whose amplitudes decrease exponentially with increasing $t$, prove to be negligible for this reason in relation to the nucleon-resonance contributions. The amplitude for diffractive rho-meson production, $A_{\text {diff }}$ (see Fig. $2 g$ ), is a free parameter of the nonresonance mechanisms in channel (3). It is determined from a fit to the entire set of measured cross sections for the reactions $\gamma r, v p$ $\rightarrow \pi+\pi-p$. The amplitudes for the channels in (1)-(3) are calculated on the basis of the model developed in [5- 8]. In doing this, use is made of the corresponding three-particle diagrams in Fig. 1. This makes it possible to obtain integrated, differential, and multiple differential (correlation) cross sections for each of the channels in (1)-(3). The aforementioned free parameters of the resonance and nonresonance mechanisms for the isobar channels (1)-(3) are determined from a fit to the measured cross sections for the reactions $\gamma r, v p \rightarrow \pi+\pi-p$. Therefore, the results found in this way for the contributions of the channels in (1)- (3) are considered as those that were extracted from experimental data. The dynamical model that was developed in [5- 8] and which is used to extract information about the channels in (1)-(3) describes the entire body of world-average data and the latest data of the CLAS Collaboration on the cross sections for double charged-pion production on a proton by photons over the kinematical region where the contribution of the channel in (3) was extracted. This gives every reason to believe that the parameterization used for the mechanisms of double charged-pion production on a proton by real and virtual photons is quite reliable in this region.

Thus, the cross sections obtained for the channels in (1)-(3) from a fit to data of the CLAS Collaboration [3] are the most reliable. Owing to comprehensively taking into account the dynamics of various mechanisms and effects of their interference, the method used in the present study to separate the contributions of different isobar channels is a step forward in relation to the previously employed naive descriptions of invariant-mass distributions of $\pi^{+} \pi^{-}$and $\pi^{-} p$ final products in terms of a set of resonance peaks and phase space. 


\section{SEPARATION OF ISOBAR CHANNELS IN CLAS DATA}

The contributions of the channels in (1)-(3) to the reactions $\gamma r, v p \rightarrow \pi^{+} \pi-p$ were determined from a fit to CLAS data [3] on the basis of an early version of the model for describing double chargedpion production [5-7]. Investigations of the channel in (3) revealed that it is necessary to modify the diffractive ansatz for nonresonance processes that was described in $[8,16]$. The required modification is discussed below.

The CLAS data reported in [3] include the differential cross sections

(i) $d \sigma / d M_{\pi^{+} \pi^{-}}$,

(ii) $d \sigma / d M_{\pi^{+}}$,

(iii) $d \sigma / d_{\Omega \pi^{-}}$,

where $M_{\pi^{+} \pi^{-}}$and $M_{\pi^{+} p}$ are the invariant masses of, respectively, the $\pi^{++\pi^{-}}$and $\pi^{+} p$ final-particle system, while $\Omega_{\pi^{-}}$is the solid angle of the emission of a negatively charged pion in the c.m. frame. The data in question were obtained over the kinematical region of the variables $W$ and $Q^{2}$ that is presented in the table.

Each bin in $W$ and $Q^{2}$ contained eight points in the variables $M_{\pi+\pi^{-}}$and $M_{\pi+p}$ and ten points in the c.m. angle $\theta_{\pi^{-}}$. In describing the above cross sections on the basis of the model developed in [58], the nucleon-resonance electromagnetic form factors were fixed at the values determined in the analysis reported in $[3,17]$. We varied only the nonresonance parameters of this model. Our procedure for fitting the data included
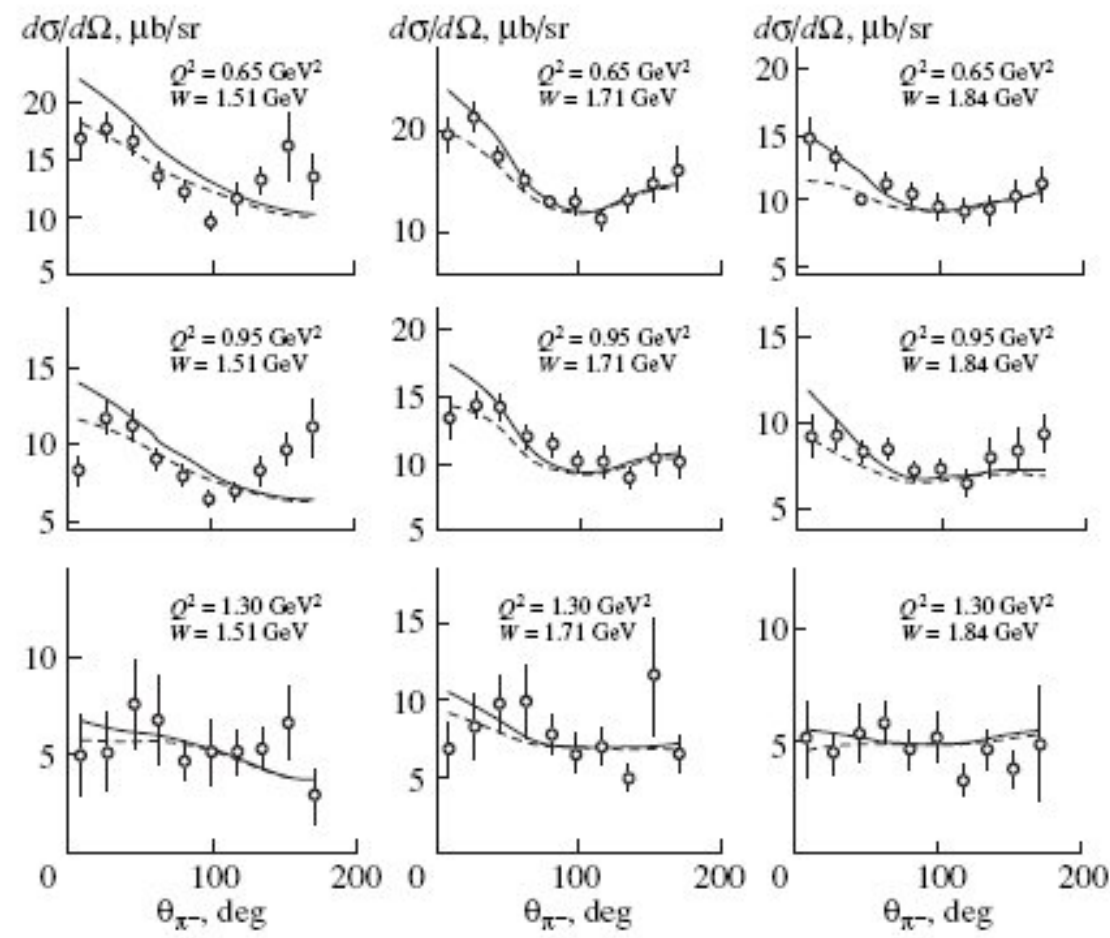

Fig. 4. Angular distributions of negatively charged pions in the c.m. frame at the same values of $Q^{2}$ and $W$ as in Fig. 3 for $\alpha_{R}$ ranging between the values indicated in (4). The displayed points represent experimental data, while the dashed and solid curves correspond to values calculated at $\alpha_{R}=1.2$ and $\alpha_{R}=1.5$, respectively.

the following steps.

First, we determined the range of the effective coupling $\alpha_{R}$ of the pion Regge trajectory to the $p \rightarrow$ $\Delta$ transition current. The coupling constant $\alpha_{R}$ was varied over a range that leads to the scatter of 
calculated cross sections in excess of the uncertainties in measured cross sections, the amplitudes of three particle phase space, $C\left(W, Q^{2}\right)$, and the amplitude of the nonresonance diffractive production of a rho meson being fixed at values obtained from an analysis reported in $[3,17]$. For $\alpha_{R}$, we determined a value that corresponds to the best description of the data and the range of values that is compatible with the uncertainties in the measured cross sections for double charged-pion production. The objective of the next step in this analysis was to determine cross sections for elastic rho-meson electroproduction on a proton in the energy region of nucleon-resonance excitations [channel (3)]. Upon fixing $\alpha_{R}$ at a value that corresponds to the best description of the data at the preceding step, we tuned the amplitudes for three-particle phase space, $C\left(W, Q^{2}\right)$. The amplitudes $C\left(W, Q^{2}\right)$ were chosen on the basis of a fit to data on the invariant masses of $\pi^{+} \pi^{-}$pairs off the rho-meson peak. After fixing the amplitudes $C\left(W, Q^{2}\right)$ in this way, we further varied the amplitudes for nonresonance diffractive rho-meson production, $A_{\text {diff }}$ We determined $A_{\text {diff }}$ from a fit to the entire body of data from [3].

By using the nonresonance parameter $A_{\text {diff }}$ obtained on the basis of the procedure outlined above, we calculated amplitudes and cross sections for the reactions $\gamma r, v p \rightarrow \pi+\pi-p$ involving the production of a rho meson in the intermediate state (see Figs. $2 f, 2 g$ ). The cross section obtained in this way was treated as the cross section for the reaction in (3).

Below, we construct fits to the CLAS data [3] for all of the steps listed above.

\subsection{Determination of the Effective Coupling $\alpha \mathrm{R}$ of the Pion Regge Trajectory to the $p \rightarrow \Delta$ Transition Current}

In determining the effective coupling $\alpha_{R}$ of the pion Regge trajectory to the $p \rightarrow \Delta$ transition current, the angular distributions of negatively charged pions prove to be the most sensitive observables. Therefore, $\alpha_{R}$ was determined from data on angular distributions of negatively charged pions for all ranges of $W$ and $Q^{2}$ presented in the table. The Reggeon coupling constant was varied between 1.1 and 1.7 around the value obtained previously in [3]. The ranges of angular distributions of negatively charged pions according to calculations with this variation of $\alpha_{R}$ are shown in Fig. 3. In the region of forward angles, where the contribution of the nonresonance mechanisms of the channel in (1) is the most pronounced, the scatter of the calculated curves covers completely the range of the measured cross sections. Thus, the range chosen for the variations in $\alpha_{R}$ contains the value that corresponds to the best description of the data. This value was determined by minimizing the $\chi^{2}$ functional calculated on the basis of a comparison of the measured angular distributions of negatively charged pions with their counterparts calculated on the basis of the model developed in [5-7]. The $\chi^{2}$ values were calculated from a comparison of experimental data and the results of the calculations in all available bins in $W$ at fixed $Q^{2}$. Minimization was performed independently in each of the three ranges in $Q^{2}$.

Further, we determined the range of $\alpha_{R}$ variations that is compatible with the uncertainties in the data. For $\alpha_{R}$, we selected values at which the calculated cross sections fall within the range of the uncertainties in the measured angular distributions. This was achieved by selecting cross sections that were calculated with varied $\alpha_{R}$ and for which $\chi^{2}<\chi^{2}$ thr, where the threshold value $\chi^{2}$ thr corresponds to the scatter of the calculated cross sections within the errors in the data. The values of $\alpha_{R}$ for the calculated cross sections that we selected were averaged.

The average value and the variance for the selected values of $\alpha_{R}$ were identified, respectively, with the mean value obtained for $\alpha_{R}$ from a fit to CLAS data from [3] and with the uncertainty in it.

The result obtained in this way for the effective coupling of the pion Regge trajectory to the $p \rightarrow \Delta$ transition current is

$$
\alpha_{R}=1.35 \pm 0.15 \text {. (4) }
$$

The scatter of the calculated cross sections that was obtained upon varying $\alpha_{R}$ over this range is displayed in Fig. 4. The calculations faithfully reproduce experimental data for $W>1.55 \mathrm{GeV}$. This is precisely the kinematical region where the data prove to be sensitive to the contribution of the 
channel in (3). At lower $W$, the cross sections for this channel are within the measurement errors. The ultimate objective of the analysis presented in this article is to extract the cross section for elastic rho-meson electroproduction on a proton.

In the region $W<1.55 \mathrm{GeV}$, the cross sections calculated for c.m. emission angles of negatively charged pions in excess of 130 o are much smaller than their measured counterparts. This drawback was overcome by refining the dynamical model for

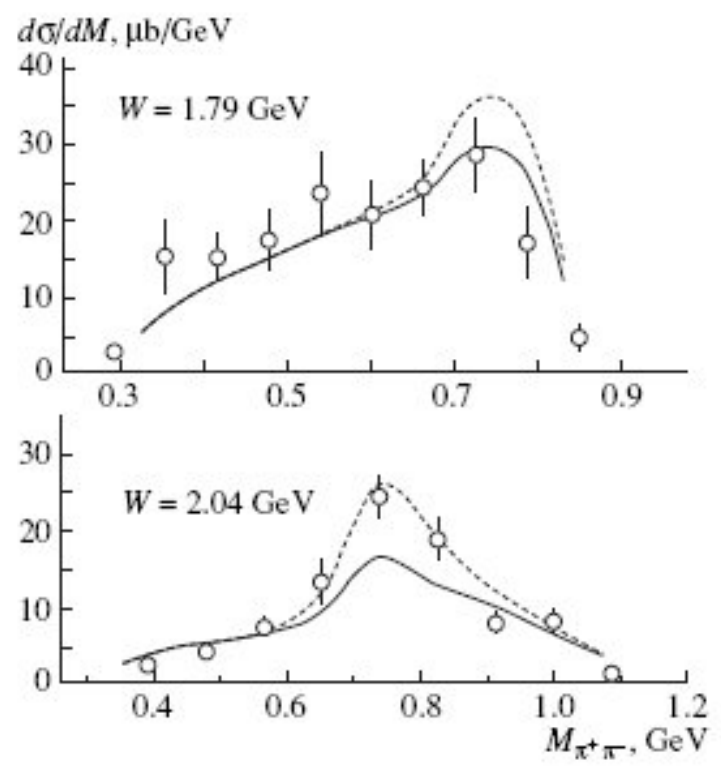

Fig. 5. Invariant-mass distribution of $\pi+\pi$ - pairs at $Q^{2}=1.3 \mathrm{GeV}^{2}$ and $W=1.79$ or $2.04 \mathrm{GeV}$. The displayed experimental data (points) were borrowed from [3]. The calculations were performed on the basis of the model developed in [5-8], the parameter Adiff being set to $A_{\text {diff }}=$ (solid curves) 3 or (dashed curves) 12.

double charged-pion production on a proton by real and virtual photons via replacing the naive parameterization of three-particle phase space by exchange processes for residual mechanisms [2]. Since the channels in (1) and (2) contribute over the entire range of $W$, the cross sections and amplitudes corresponding to them can be extracted only within the dynamical-model version refined according to $[1,2]$. This will

Kinematical regions of the variables $W$ and $Q^{2}$ where the cross sections for double charged-pion production by virtual photons were measured [3] (data of the CLAS Collaboration) [in the left column, we present the boundaries of the intervals in $Q^{2}$ (upper rows) and the values of $Q^{2}$ at which fits to data were constructed]

\begin{tabular}{c|c|c}
\hline$Q^{2}, \mathrm{GeV}^{2}$ & $\begin{array}{c}W \\
\text { range, } \mathrm{GeV}\end{array}$ & $\begin{array}{c}\text { Number of ranges in } \\
W\end{array}$ \\
\hline $0.5-0.8$ & $1.41-1.89$ & 20 \\
0.65 & & \\
$0.8-1.1$ & $1.41-1.89$ & 20 \\
0.95 & & \\
$1.1-1.5$ & $1.41-2.10$ & 28 \\
1.30 & & \\
\hline
\end{tabular}



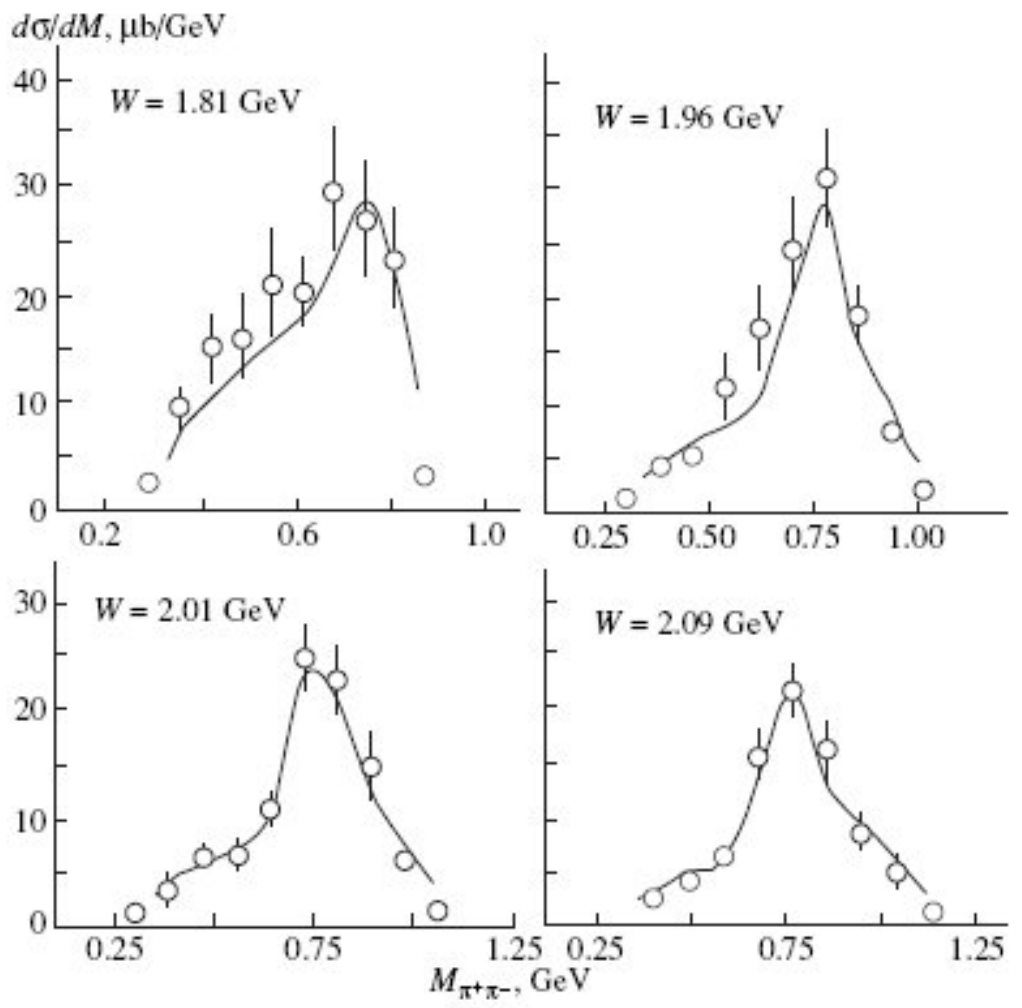

Fig. 6. Invariant-mass distribution of $\pi+\pi$ - pairs at $Q^{2}=1.30 \mathrm{GeV}^{2}$ upon introducing the parameterization according to (5)-(7) for $A_{\text {diff }}(W)$. The points represent experimental data from [3].

be the subject matter of our forthcoming publication.

\subsection{Analysis of Processes Involving Elastic Rho-Meson Electroproduction}

The channel of elastic rho-meson electroproduction manifests itself in (i) invariant-mass distributions of $\pi+\pi-$ pairs and (ii) angular distributions of negatively charged pions. In the energy region of nucleon resonance excitation, the contribution of the channel in (3) is much less than the contribution of the channel in (1). Therefore, the invariant-mass distributions of $\pi^{+} \pi^{-}$pairs, where the signal from the channel in (3) has the shape of a peak at the $\pi^{+} \pi^{-}$mass equal to the rho-meson mass, are the most sensitive to elastic rho-meson electroproduction. In order to determine the contributions from the channel in (3), we analyzed invariant-mass distributions of $\pi+\pi$ - pairs.

The effective coupling $\alpha_{R}$ of the pion Regge trajectory to the $p \rightarrow \Delta$ transition current was fixed at a value that corresponds to the best description of angular distributions of negatively charged pions in the c.m. frame. The amplitudes for three-particle phase space were additionally tuned in order to ensure the best description of data on the invariant masses of $\pi^{+} \pi^{-}$pairs off the rhomeson peak. An investigation of elastic rho-meson photo- and electroproduction at invariant masses of the final hadron system in excess of energies of nucleon-resonance excitation $(W>2.0$ $\mathrm{GeV})[16,18]$ revealed that the constant $A_{\text {diff }}$ for the nonresonance diffractive production of a rho meson is independent of $W$. In analyzing CLAS data [3] on invariant-mass distributions of the $\pi^{+} \pi^{-}$final-particle system, we found that, in the region $W<2.1 \mathrm{GeV}$, the amplitude for diffractive rho-meson production is dependent on $W$. Figure 5 shows fits to invariant-mass distributions of $\pi^{+} \pi^{-}$pairs at $W=1.79$ and $2.04 \mathrm{GeV}$ and $Q 2=1.3 \mathrm{GeV} 2$ for two markedly different values of the amplitude: $A_{\text {diff }}=3$ and 12. From a comparison of the results of the calculations and the experimental data in Fig. 5, it can clearly be seen that a good description of invariant-mass distributions of $\pi^{+} \pi^{-}$pairs at $W=1.79 \mathrm{GeV}$ is attained at $A_{\text {diff }}=3$. At this value of $A_{\text {diff, }}$ the 
contribution of the channel in (3) proves to be underestimated, however, at $W=2.04 \mathrm{GeV}$. The increase in the parameter $A_{\text {diff }}$ from 3 to 12 makes it possible to describe well data at $W=2.04$ $\mathrm{GeV}$, but this leads to a pronounced overestimation of the channel
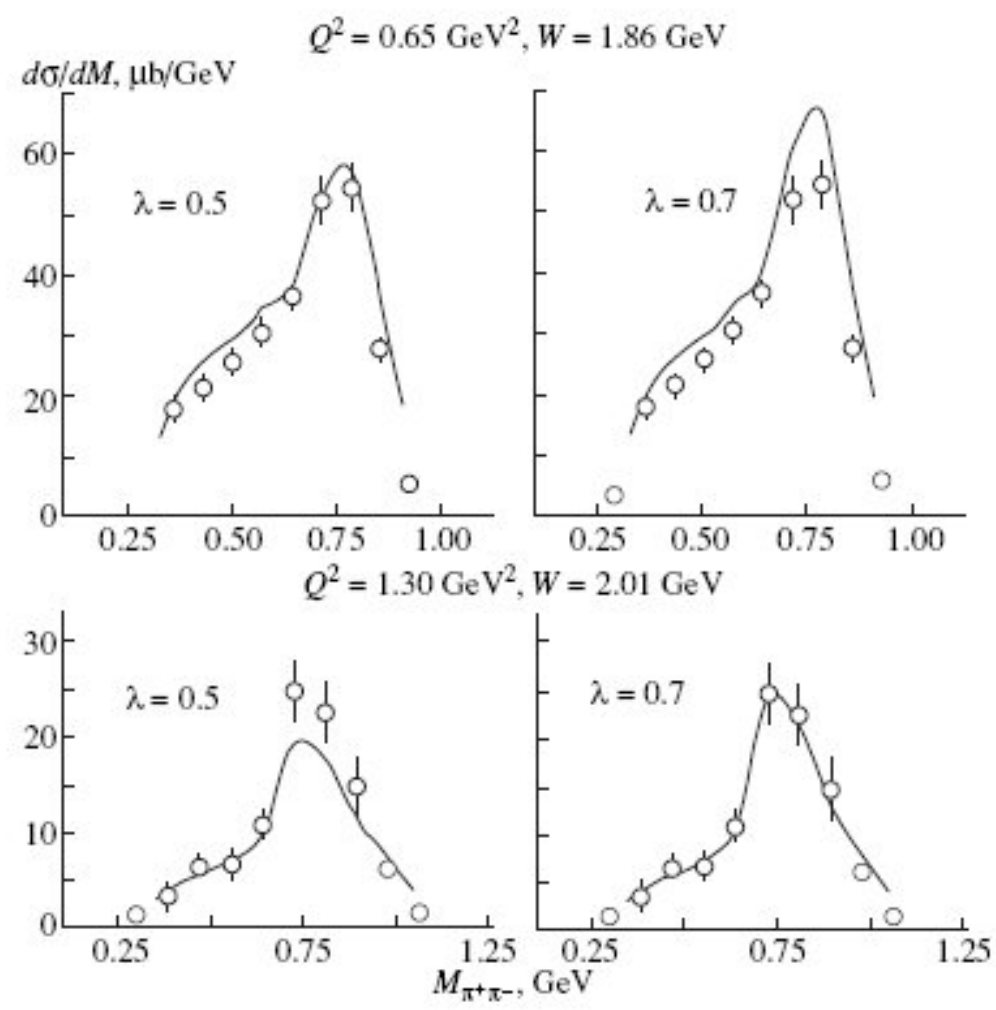

Fig. 7. Invariant-mass distribution of $\pi+\pi$-pairs at $Q_{2}=0.65$ and $1.30 \mathrm{GeV}_{2}$ upon introducing the parameterization according to (5)-(8) for $A_{\text {diff }}(W)$ at various fixed values of the parameter $\lambda$. The points represent experimental data from [3].

in (3) at $W=1.79 \mathrm{GeV}$. Thus, the CLAS data from [3] on invariant-mass distributions of $\pi^{+} \pi^{-}$pairs suggest that $A_{\text {diff }}$ depends on $W$ in the energy region of nucleon-resonance excitation.

This dependence may arise because of the proximity to the rho-meson-production threshold, which is $1.71 \mathrm{GeV}$ at the central value of the rho-meson mass. At the reaction threshold, the reaction amplitude must vanish. This behavior of the amplitudes must lead to a reduction of $A_{\text {diff }}$ with decreasing $W$. Here, it is necessary to take into account the fact that the rho meson is an unstable particle of decay width $150 \mathrm{MeV}$. A finite width of the rho meson leads to a nonzero cross section for its production in the sub-threshold region $(W<1.71 \mathrm{GeV})$ owing to the population of part of the $\rho$-meson line in the region of low values of the $\pi^{+} \pi^{-}$invariant mass. We parameterized the $W$ dependence of $A_{\text {diff }}$ by an exponential function that is saturated in the region $W>2.0 \mathrm{GeV}$ and which vanishes at $W=1.41 \mathrm{GeV}$ (the production threshold minus the rho-meson decay width according to the calculation at the central value of the rho-meson mass); that is,

$$
A_{\text {diff }}(W)=A_{0}(1-\exp (-(W-1.41) / D)),(5)
$$

where $A_{0}$ and $D$ are phenomenological parameters and $W$ is measured in $\mathrm{GeV}$ units.

This parameterization approximately corresponds to $W$-independent $A_{\text {diff }}$ in the region $W>2.5$ $\mathrm{GeV}$, in agreement with data on rho-meson photo- and electroproduction in this kinematical region [16, 18]. As $W$ becomes lower, the amplitude decreases, vanishing at $W$ equal to the central value of the rho-meson mass minus the rho-meson decay width. The parameters $A_{0}$ and $D$ were determined from a fit to CLAS data [3] on invariant-mass distributions of $\pi^{+} \pi^{-}$pairs at $Q^{2}$ $=1.30 \mathrm{GeV} 2$. The ranges of the parameters $A_{0}$ and $D$ were such that cross sections calculated 
with parameters varied within these ranges were scattered within limits covering CLAS data from [3]. The procedure for determining $A_{0}$ and $D$ values compatible with these data is identical to that which was described in Subsection 3.1 for the parameter $\alpha_{R}$. The values extracted for the parameters $A_{0}$ and $D$ from our fit to the data are

$$
\begin{gathered}
A_{0}=12 \pm 1,(6) \\
D=0.25 \pm 0.1 \mathrm{GeV} .(7)
\end{gathered}
$$

The invariant-mass distributions of $\pi^{+} \pi^{-}$pairs according to calculations on the basis of the model developed in [5-8] are presented in Fig. 6 along with their counterparts

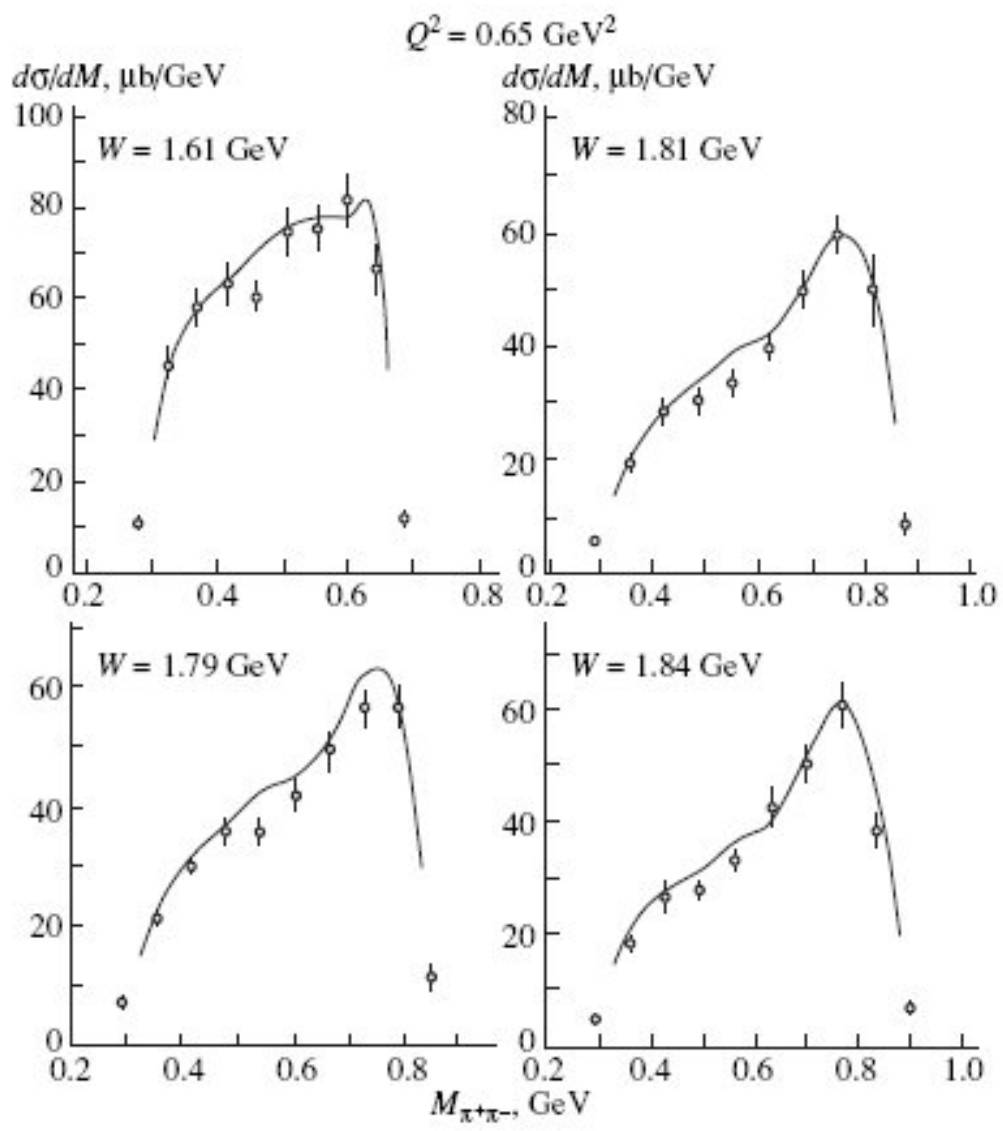

Fig. 8. Invariant-mass distribution of $\pi+\pi$-pairs at $Q^{2}=0.65,0.95$, and $1.3 \mathrm{GeV}^{2}$ within the modification proposed in this study for the nonresonance part of the channel in (3). The points represent experimental data from [3].

measured by the CLAS Collaboration. At $Q^{2}=1.30 \mathrm{GeV}^{2}$, the results of the calculations are given for $A_{0}$ and $D$ values that correspond to the best description of CLAS data from [3]. At this value of $Q^{2}$, a good description of these data is attained over the entire range of $W$ values that is covered by the CLAS measurements reported in [3]. The CLAS data also proved the need for modifying the description of the $Q^{2}$ evolution of the amplitude for diffractive rho-meson production, $A_{\text {diff }}\left(Q^{2}\right)$. Within the model developed in [5-8], the $Q^{2}$ evolution of $A_{\text {diff }}$ is described on the basis of the vector-meson dominance concept, which was used in [16]; that is,

$$
A_{\text {diff }}\left(Q^{2}\right) \sim\left(1+Q^{2} / \lambda^{2}\right)^{-1} \text {. }
$$

In Fig. 7, the results of the calculations from [5- 8] are contrasted against the CLAS data from [3] on invariant-mass distributions of $\pi^{+} \pi^{-}$pairs at $Q^{2}=0.65$ and $1.30 \mathrm{GeV}^{2}$. The calculations were performed at two values of the cutoff parameter $\lambda$ in (8): $0.7 \mathrm{GeV}$ (which is a nominal value within the model developed in [5-8]) and $0.5 \mathrm{GeV}$. From the data in Fig. 7, it follows that, upon the 
introduction of the $W$-dependent amplitude $A_{\text {diff }}(W)$, the model in question is unable to reproduce the data at the minimum and maximum values of $Q^{2}$ in the CLAS measurements reported in [3]. The parameter value of $\lambda=0.7 \mathrm{GeV}$, which corresponds to the best description of the CLAS data at $Q^{2}=1.30 \mathrm{GeV}^{2}$, leads to overestimating the contribution of the channel in (3) at $Q^{2}=0.65$ $\mathrm{GeV}^{2}$. In order to reproduce the CLAS data at $Q^{2}=0.65 \mathrm{GeV}^{2}$, the parameter $\lambda$ must be reduced to $0.5 \mathrm{GeV}$, but, at $\lambda=0.5 \mathrm{GeV}$, the contribution of the channel in (3) at $Q^{2}=1.30 \mathrm{GeV}^{2}$ and $W=$ $2.01 \mathrm{GeV}$ proves to be underestimated. Thus, the CLAS data are indicative of the need for introducing in (8) a $W$-dependent cutoff parameter $\lambda$.

The parameterization of the $Q^{2}$ dependence of $A_{\text {diff }}$ in (8) corresponds to a transition between a photon and a rho meson, as is expected in the vector-meson dominance model. At $W$ values near the rho-meson production threshold, only part of the rho-meson line may prove to be on-shell, this giving rise to a $W$ dependence in $\lambda$.

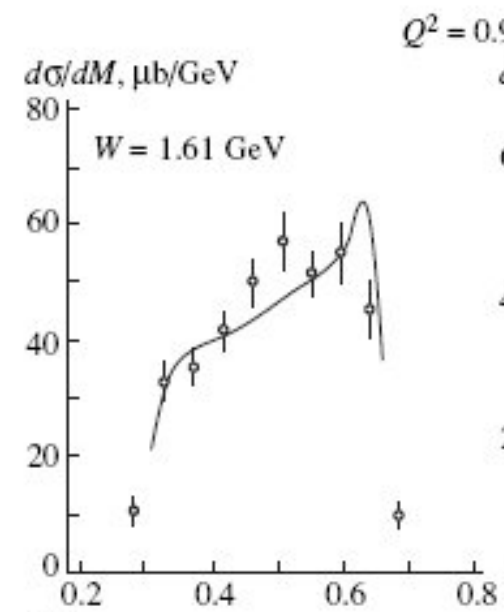

$Q^{2}=0.95 \mathrm{GeV}^{2}$
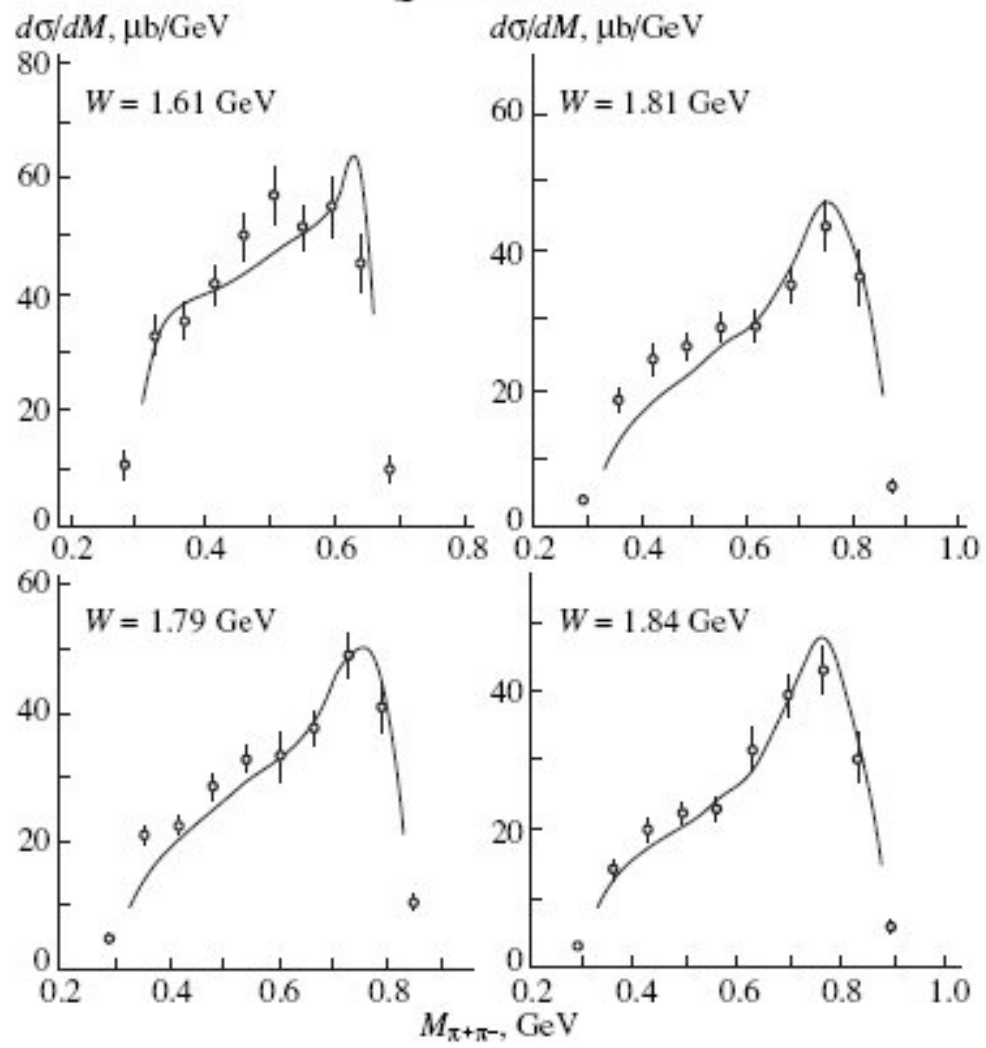

Fig. 8. (Contd.)

The $W$ dependence of the cutoff parameter $\lambda(W)$ was parameterized in terms of an exponential function that is saturated in the region $W>1.75 \mathrm{GeV}$, in which case the rho-meson line is entirely produced on the mass shell:

$$
\lambda(W)=\lambda_{0}\left(1-\exp (W-1.41) / D_{\lambda}\right) .(9)
$$

Substituting (8) and (9) into (5), we find that the dependence of $A_{\text {diff }}\left(Q^{2}, W\right)$ on the photon virtuality $Q^{2}$ and the invariant mass $W$ of the final hadron system has the form

$$
\begin{gathered}
A\left(W, Q^{2}\right)=A_{0}(1-\exp (-(W-1.41) / D))(10) \\
\times\left[\left(1+Q^{2} / 0.5\right) /\left(1+Q^{2} / \lambda^{2}(W)\right)\right],
\end{gathered}
$$

where $Q^{2}$ and $W$ are measured in, respectively, $\mathrm{GeV}^{2}$ and $\mathrm{GeV}$ units. The numerator in the second factor on the right-hand side of $(10)$ is a normalization factor. It corresponds to the $Q^{2}$ 
evolution of $A_{\text {diff }}$ at a nominal parameter $\lambda$ that is independent of $W$ and which was used in [9]. On the basis of the parameterization in (10) for $A_{\text {diff, }}$ we constructed a fit to invariant mass distributions of $\pi+\pi$ - pairs over the entire range of $Q^{2}$ and $W$ that is covered by the CLAS measurements reported in [3]. The quantities $\lambda_{0}$ and $D_{\lambda}$ in (9) were treated as free parameters. Their values determined from a fit to the data are

$$
\begin{aligned}
& \lambda_{0}=0.77 \mathrm{GeV}, \\
& D_{\lambda}=0.3 \mathrm{GeV} .
\end{aligned}
$$

Figure 8 shows the calculated and measured invariant mass distributions of $\pi+\pi$ - pairs in the kinematical region of the variables $W$ and $Q^{2}$ that is covered by the CLAS measurements reported in [3]. The modification developed in the present study for the nonresonance part of the amplitude for diffractive rho meson production [see Eq. (10)] made it possible to describe well all available CLAS data on the reactions $\gamma r, v p \rightarrow \pi+\pi-p$ in the region $W>1.55 \mathrm{GeV}$. Thus, this method enables one to extract reliably cross sections and amplitudes for the channel in (3) from the differential cross sections for double charged-pion production on a proton by virtual photons.
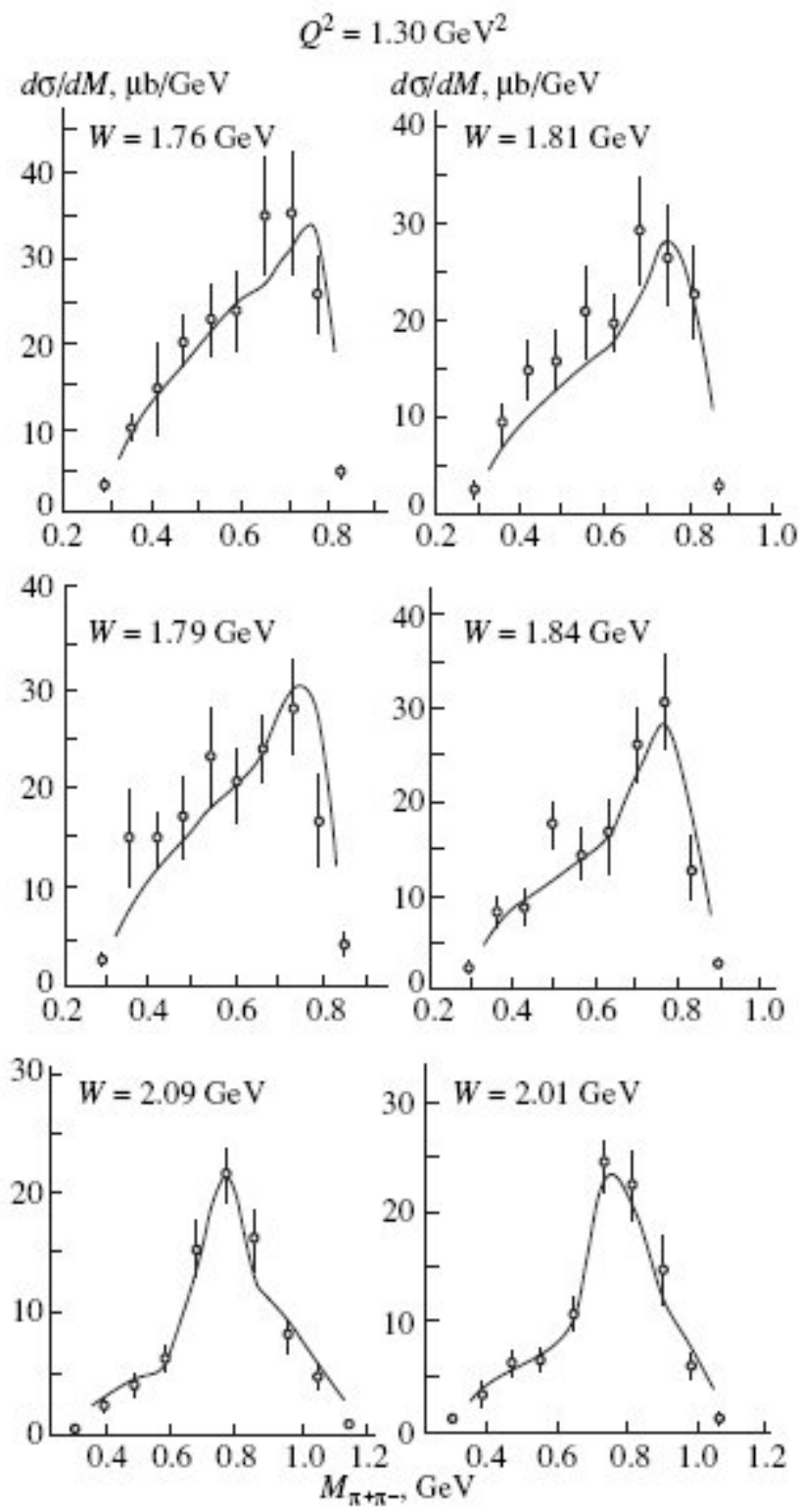
Fig. 8. (Contd.)

\section{CROSS SECTIONS FOR ELASTIC RHO-MESON ELECTROPRODUCTION ON A PROTON IN THE ENERGY REGION OF NUCLEON-RESONANCE EXCITATION}

From the fit to CLAS data [3] that was described above, we have determined the amplitude $A_{\text {diff }}\left(W, Q^{2}\right)$, which is necessary for calculating the nonresonance part of the amplitude for the channel in (3). The nucleon-resonance parameters were borrowed from the analysis reported in $[3,17]$. Thereby, we have determined all of the parameters that are necessary for evaluating the amplitudes (see Figs. $2 f, 2 g$ ) and cross sections for elastic rho-meson electroproduction on a proton. The range of cross sections for the channel in (3) according to the fit to the CLAS data reported in [3] is displayed in Fig. 9. The uncertainties in the cross section for elastic rho-meson electroproduction on a proton correspond to fluctuations of the parameters of $A_{\text {diff }}\left(W, Q^{2}\right)$ within the limits indicated in Eqs. (6) and (7). Since, after the above modifications in the description of nonresonance $\rho$-meson production, the model developed in [5-8] faithfully reproduces the entire body of CLAS data from [3] on double pion electroproduction, the cross sections extracted within our approach for the isobar channel (3) are quite reliable. The contributions of isobar channels were isolated previously by fitting invariant mass

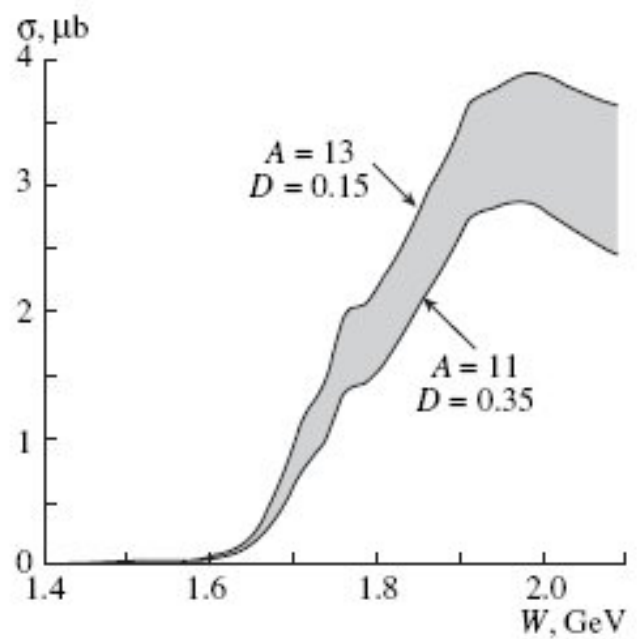

Fig. 9. Integrated cross section for the reactions $y r, v p \rightarrow \rho p$ at $Q_{2}=1.30 \mathrm{GeV}_{2}$ according to data of the CLAS Collaboration [3].

distributions of $\pi^{+} \pi^{-}$and $\pi^{+} p$ pairs in terms of a combination of the phase space and resonance curves corresponding to the formation of unstable particles in the final state. The approach proposed here is advantageous in that it (i) enables us to analyze various measured cross sections (angular and invariant-mass distributions), ensuring the respective reflection of isobar channels; (ii) takes into account effects of channel interference; and (iii) permits determining, along with integrated cross sections for isobar channels, their differential cross sections. The cross sections obtained for elastic rho-meson electroproduction within the approach developed here are unique data in the world on this isobar channel in the energy region of nucleonresonance excitation in reactions involving virtual photons.

The range of $Q^{2}$ between 1.1 and $1.5 \mathrm{GeV}^{2}$ corresponds to the maximum range of $W$ values that is covered in the CLAS measurements, from the threshold to $2.1 \mathrm{GeV}$. In the region $W>1.9 \mathrm{GeV}$, the CLAS data ensure a reliable selection of the rho-meson peak. The requirement that the threeparticle phase space depend smoothly on $W$ makes it possible to separate the rho-meson peak from the phase space in the CLAS data at $Q^{2}=1.30 \mathrm{GeV}^{2}$ for $W<1.9 \mathrm{GeV}$ inclusive. 
In the region $Q^{2}<1.1 \mathrm{GeV}^{2}$, the CLAS measurements cover the range $W<1.9 \mathrm{GeV}$ (see table). This circumstance leads to additional ambiguities in separating the rho-meson peak from the three particle phase space. In order to separate reliably these contributions, it is necessary to analyze a wider database, including the entire body of measured cross sections. Such an analysis can be performed only on the basis of the latest, substantially refined, version of our model (this version was discussed elsewhere [1, 2]). At the present time, this work is under way, and its results will be the subject matter of our subsequent publications.

\section{CONCLUSIONS}

We have developed a method for determining the contribution of various isobar channels to the reactions $\gamma r, v p \rightarrow \pi+\pi-p$. We have relied on the model developed in [5-8] for describing double charged pion production on a proton by real and virtual photons. We have determined phenomenological parameters of the model from a fit to the measured cross sections for the reactions $\gamma r, v p \rightarrow \pi+\pi-p$. By using parameters evaluated in this way, we have calculated amplitudes and cross sections for isobar channels. Thereby, we have deduced the entire body of information about the contributions of isobar channels. Our analysis of the latest data of the CLAS Collaboration on the reactions $\gamma r, v p \rightarrow \pi+\pi-p$ near the threshold for rho-meson production has revealed the dependence of the amplitudes for the nonresonance diffractive production of rho mesons on the kinematical variables $W$ and $Q^{2}$. In this study, we have developed an approach to describing the dependence of the diffraction amplitude on these variables. A modification of the diffractive ansatz has made it possible to reproduce faithfully CLAS data on double charged pion electroproduction on a proton in the kinematical region corresponding to the contribution of the channel in (3).

The integrated cross sections for the reactions $\gamma r, v p \rightarrow \rho p$ at energies of nucleon-resonance excitation have been determined for the first time on the basis of the approach developed here, this being done in the near-threshold and subthreshold kinematical regions inclusive.

\section{REFERENCES}

1. V. D. Burkert and T.-S. H. Lee, Int. J. Mod. Phys. E 13, 1035 (2004).

2. V. D. Burkert, V. I. Mokeev, M. Ripani, et al., in Proceedings of the Workshop on the Physics of Excited Nucleons, Grenoble, France, 2004, p. 317.

3. M. Ripani, V. D. Burkert, V. I. Mokeev, et al., Phys.Rev. Lett. 91, 022002 (2003).

4. M. Bellis, in Proceedings of the Workshop on the Physics of Excited Nucleons, Grenoble, 2004, p. 187.

5. M. Ripani, V. I. Mokeev, M. Anghinolfi, et al., Nucl.Phys. A 672, 220 (2000).

6. M. Ripani, V. I. Mokeev, M. Battaglieri, et al., Yad.Fiz. 63, 2036 (2000) [Phys. At. Nucl. 63, $1943(2000)]$.

7. V. I. Mokeev,M.Ripani, M. Anghinolfi, et al., Yad. Fiz.64, 1368 (2001) [Phys. At. Nucl. 64, 1292 (2001)].

8. V. D. Burkert,V. I. Mokeev,G. V. Fedotov, et al.,Nucl. Phys. A 737, S231 (2004).

9. T. D. Cohen, D. C. Dakin, R. F. Lebed, andD.R.Martin, Phys. Rev. D 71, 076010 (2005).

10. T. P. Vrana, S. A. Dytman, and T.-S. H. Lee, Phys. Rep. 328, 181 (2000).

11. ABBHHM Collab., Phys. Rev. 175, 1669 (1968).

12. K.Wacker et al., Nucl. Phys. B 144, 269 (1978).

13. V. D. Burkert, V. I. Mokeev,M. Ripani, et al., Yad. Fiz. 66, 2199 (2003) [Phys. At. Nucl. 66, 2149 (2003)].

14. D. M. Manley and E. M. Saleski, Phys. Rev. D 45, 4002 (1992).

15. M. Guidal, J.-M. Laget, and M. Vanderhaeghen, Phys. Lett. B 400, 6 (1997).

16. D. G. Cassel et al., Phys. Rev. D 24, 2787 (1981).

17. M. Anghinolfi, M. Battaglieri, E.N.Golovach, et al., Yad. Fiz. 62, 1522 (1999) [Phys. At. Nucl. 62, 1448 (1999)].

18. M. Battaglieri, E. Anciant,M. Anghinolfi, et al.,Phys. Rev. Lett. 87, 172002 (2001).

Translated by A. Isaakyan 